\title{
Band dispersion of graphene with structural defects
}

\author{
Piotr Kot, ${ }^{1}$ Jonathan Parnell, ${ }^{2}$ Sina Habibian, ${ }^{2}$ Carola Straßer, ${ }^{1}$ Pavel M. Ostrovsky, ${ }^{1,3}$ and Christian R. Ast $\circledast^{1, *}$ \\ ${ }^{1}$ Max-Planck-Institut für Festkörperforschung, 70569 Stuttgart, Germany \\ ${ }^{2}$ University of British Columbia, Vancouver, Canada \\ ${ }^{3}$ L. D. Landau Institute for Theoretical Physics RAS, 119334 Moscow, Russia
}

(Received 2 November 2018; revised manuscript received 28 April 2020; accepted 5 May 2020; published 3 June 2020)

\begin{abstract}
We study the band dispersion of graphene with randomly distributed structural defects using two complementary methods, exact diagonalization of the tight-binding Hamiltonian and implementing a self-consistent $T$ matrix approximation. We identify three distinct types of impurities resulting in qualitatively different spectra in the vicinity of the Dirac point. First, resonant impurities, such as vacancies or 585 defects, lead to stretching of the spectrum at the Dirac point with a finite density of localized states. This type of spectrum has been observed in epitaxial graphene by photoemission spectroscopy and discussed extensively in the literature. Second, nonresonant (weak) impurities, such as paired vacancies or Stone-Wales defects, do not stretch the spectrum but provide a line broadening that increases with energy. Finally, disorder that breaks sublattice symmetry, such as vacancies placed in only one sublattice, open a gap around the Dirac point and create an impurity band in the middle of this gap. We find good agreement between the results of the two methods and also with the experimentally measured spectra.
\end{abstract}

DOI: 10.1103/PhysRevB.101.235116

\section{INTRODUCTION}

Graphene has presented high potential for providing the next generation of electronic materials due to its strictly two-dimensional character as well as its high electron mobility. It has demonstrated high design flexibility, such as doping by atoms or molecules, efficient decoupling from an underlying substrate, or high tensile strength for flexible electronics [1-3], and has been the catalyst for the creation of new fields of study such as two-dimensional materials or Dirac semimetals [4-10]. Several theoretical proposals as well as experiments are concerned with enhancing the spin-orbit coupling to open a band gap or inducing spin splitting [11,12]. Even a transition to a superconducting state has been theoretically and experimentally demonstrated [13]. However, one of the most important prerequisites for graphene, and other twodimensional materials, to become a base material for future electronics concerns the opening of a band gap, which has not been successfully demonstrated so far. For graphene the linear crossing of the bands near the Dirac point is protected by symmetry, because the two sublattices are equivalent. In order to open a band gap, this sublattice symmetry has to be broken.

Angle-resolved photoemission spectroscopy (ARPES) is the most direct method to probe the electronic structure

\footnotetext{
${ }^{*}$ Corresponding author: c.ast@ fkf.mpg.de
}

Published by the American Physical Society under the terms of the Creative Commons Attribution 4.0 International license. Further distribution of this work must maintain attribution to the author(s) and the published article's title, journal citation, and DOI. Open access publication funded by the Max Planck Society. experimentally. Numerous studies have examined the band structure of graphene near the Dirac point [14-23]. Several of these studies observe an elongated region near the Dirac point as if the two touching cones are pulled apart, stretched but without tearing apart. Such occurrences have been discussed extensively in literature and, depending on the specific environment of the graphene, were attributed to imperfections in the graphene, interactions with the substrate, or the opening of a band gap [23-28]. One observation common to all instances of the stretched Dirac point is the residual spectral weight that is still present at lowest energies. It needs to be understood in more detail in order to judge if and under what conditions it can be referred to as an actual gap.

In this paper, we present a real-space tight-binding calculation modeling different kinds of structural defects randomly distributed over a graphene sample. We show that in all instances, except the case of vacancies placed in a single sublattice, there is no band gap opening near the Dirac point. The spectrum near the Dirac point is either almost unchanged or exhibits a stretched Dirac point with broadened states, which resemble experimentally observed band dispersion. Complementing our tight-binding model with a self-consistent $T$ matrix approximation (SCTMA) calculation [29-31], we show that there are two types of point defects in graphene, resonant or nonresonant $[32,33]$. This not only provides insight into the possible origin of the "elongated" Dirac point, but also gives a way to categorize point defects. It is resonant defects that produce a dispersion with broadened states near the Dirac point resembling the results of the tight-binding calculation as well as experimental findings. Due to the remarkable similarity between the results of the SCTMA, numerical simulations of the tight-binding model, and the experiment, we conclude that the broadened states measured in epitaxial graphene must at 
least in part be caused by resonant defects in graphene. On the other hand, nonresonant defects are shown to weakly affect the dispersion near the Dirac point, which is also confirmed by the tight-binding model calculation. Although we may expect similar results for the tight-binding model and the SCTMA, because both calculations start with a real-space tight-binding defect Hamiltonian, this is not necessarily the case, as our tight-binding model considers higher orders of nearest-neighbor hopping. The similarity in the results shows that the approximation in the SCTMA is valid. Our findings lead to the conclusion that it is close to impossible to open a band gap in graphene by virtue of defects only.

\section{METHODOLOGY}

We employed a second nearest-neighbor real-space tightbinding model with the following Hamiltonian:

$$
H=-\sum_{\langle i j\rangle}|i\rangle t\left\langle j\left|+\sum_{\langle i j\rangle\rangle}\right| i\right\rangle t^{\prime}\langle j| .
$$

Here the indices $i$ and $j$ label individual atoms with one $p_{z}$ orbital per atom. The parameters $t$ and $t^{\prime}$ are hopping amplitudes between first and second nearest neighbors, respectively. The sums with single and double angular brackets run over first and second nearest neighbors, respectively. We have typically built the Hamiltonian for a rectangular supercell of 160000 carbon atoms with periodic boundary conditions and add randomly distributed structural defects with a given concentration. The Hamiltonian is then exactly diagonalized numerically and the resulting real-space wave functions are converted to momentum space. This yields the complete set of eigenenergies $E_{n}$ and corresponding eigenfunctions $\psi_{n}(\mathbf{p})$. The spectral weight function is then computed as

$$
A(E, \mathbf{p})=\sum_{n}\left|\psi_{n}(\mathbf{p})\right|^{2} \delta\left(E-E_{n}\right) .
$$

The code was programed in Matlab using the built-in routines for calculating eigenvalues and eigenvectors as well as Fourier transforms. The defects were introduced by removing the hopping between particular randomly chosen lattice sites. The atom positions of the defects have not been relaxed, except in the case of the Stone-Wales (SW) defect, which involves repositioning of two carbon atoms [34]. The hopping terms near the defects have not been relaxed. The concentration of defects, $n_{\text {imp }}$, is the ratio of the number of carbon atoms taken out or displaced to the total number of carbon atoms in the lattice. The large number of lattice sites used in the calculation is necessary for statistical reasons to increase the number of eigenstates in the region of low density of states (DOS) near the Dirac point as well as to have a large enough number of randomly distributed defects in the supercell. For the calculations presented in this paper the values of the parameters are $t=3.033 \mathrm{eV}$ and $t^{\prime}=0.2 \mathrm{eV}$ [35].

In the alternative SCTMA calculation, we start with the exact Green's function on a honeycomb lattice at zero energy. Only nearest-neighbor hopping terms are taken into account with $t=3.033 \mathrm{eV}$. Any individual structural defect considered in our calculation perturbs at most six neighboring sites of the lattice; hence we describe it with an exact $6 \times 6 \mathrm{~T}$ matrix. We use this as the starting point of the calculation as the zero-energy $T$ matrix, which is located at the energy of the Dirac point, can be calculated exactly, allowing us to later apply an energy dependent perturbation. We convert the exact zero-energy $T$ matrix to the basis of four-component spinors governed by the continuous low-energy massless Dirac Hamiltonian with two valleys [36]. The converted $T$ matrix is averaged over all possible positions and orientations of the defect and a nonzero energy is introduced as a perturbation. The averaged Green's functions of graphene with a finite concentration of defects, $n_{\text {imp }}$, acquire the selfenergy $\Sigma=\left(n_{\text {imp }} / A\right)\langle T(E-\Sigma)\rangle$. Here we have also included the same self-energy in the argument of the impurity $T$ matrix, thus introducing the self-consistency equation. The spectral weight is calculated from the self-energy as

$$
A(E, \mathbf{p})=-\frac{2}{\pi} \operatorname{Im}\left[\frac{1}{E-\Sigma-v p}+\frac{1}{E-\Sigma+v p}\right]
$$

and is compared to the results of the tight-binding model and to the experimentally measured dispersion. Similar calculations have been performed recently showing consistent results with ours, with discrepancies that can be accounted for by our first nearest-neighbor approximation in the SCTMA calculation [37]. Further details of the SCTMA calculation can be found in the Supplemental Material [30].

\section{RESULTS}

We consider five different types of structural defects illustrated in Fig. 1. They show the effect of sublattice symmetry breaking and the qualitative difference between resonant and nonresonant defects. The first three types of impurities are vacancies that are either distributed in a single (A) sublattice, equally in both sublattices (A and B), or paired (whole $A B$ unit cells removed). These defects are shown in Figs. 1(a)$1(\mathrm{c})$. While vacancies are not feasible in graphene, they provide a good model for adatoms attached to individual lattice sites inducing a strong on-site potential [32,38]. Vacancies allow us to probe sublattice symmetry breaking and to demonstrate its effect on the band dispersion [33,36,38-40]. We also consider two other structural point defects, that have been experimentally observed in epitaxial graphene [34,41] and are shown in Figs. 1(d) and 1(e). Although other point defects are also observed, these two have been chosen as they highlight the difference between resonant and nonresonant defects. The 585 defect, Fig. 1(d), is similar to the AB paired vacancy but with two reconstructed bonds. The SW defect is shown in Fig. 1(e) and involves a $90^{\circ}$ rotation of a bond between two adjacent atoms, along with the rearranging of the hopping terms around them.

Numerically computed spectra for distributions of vacancies are shown in Figs. 1(f)-1(h). We see that vacancies placed in only one sublattice, Fig. 1(f), open a gap in the Dirac spectrum with an additional midgap band [40]. We will discuss the origin of this extra band below. Removing atoms randomly from both sublattices, as shown in Fig. 1(g), induces additional spectral weight in the vicinity of the Dirac point with momentum broadening that gets stronger closer to zero energy. At higher energies we see a band structure resembling elongated Dirac points. Finally, removing adjacent 

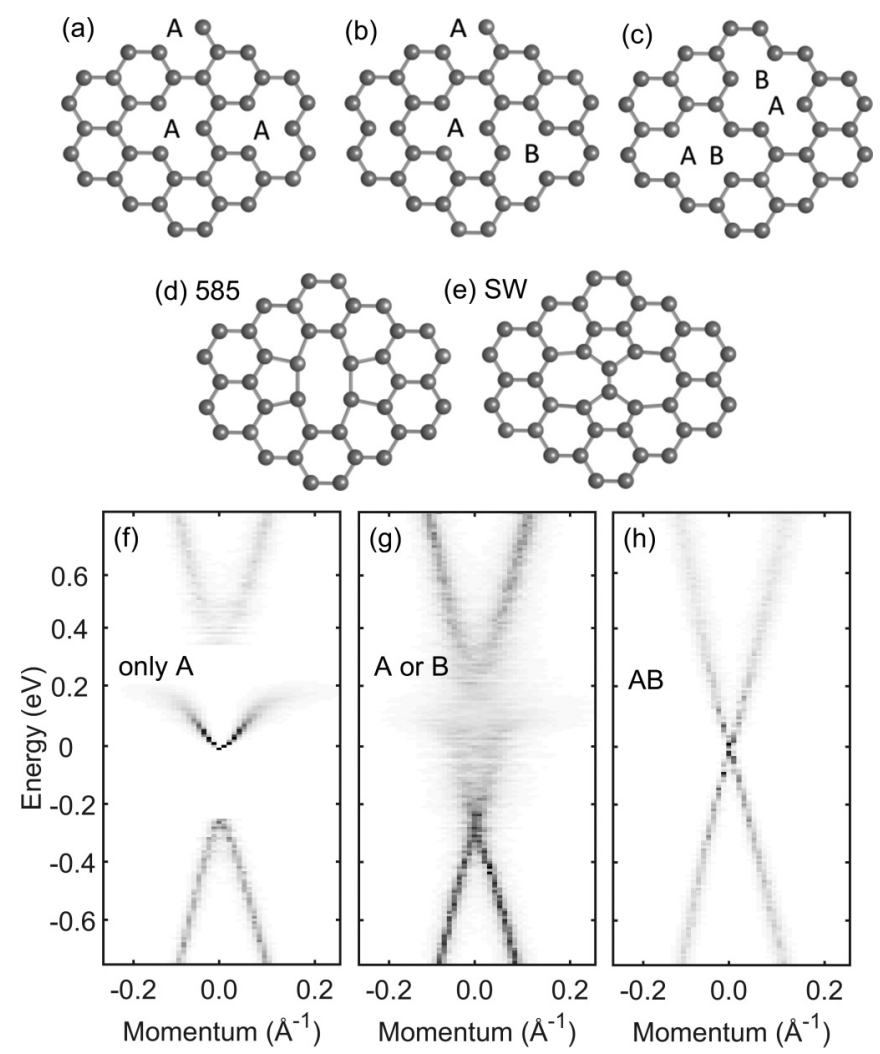

FIG. 1. Different types of structural defects: (a) vacancies in a single sublattice, (b) vacancies in both sublattices, (c) double vacancies, (d) 585 defect with two sites removed and two bonds reconstructed, and (e) Stone-Wales defect with two adjacent atoms rotated by $90^{\circ}$ [34]. Electron spectrum computed from exact diagonalization of the tight-binding model (1\% vacancy concentration): (f) for vacancies in a single sublattice, ( $g$ ) for vacancies in both sublattices, and (h) for double vacancies.

atoms, Fig. 1(h), does not noticeably affect the spectrum apart from an energy dependent broadening of the linewidth. Among these three qualitatively different electronic spectra, only the first one [vacancies placed in one sublattice, Fig. 1(a)] provides a true band gap, Fig. 1(f).

The structural defects shown in Figs. 1(d) and 1(e) also demonstrate qualitatively different spectra similar to Figs. $1(\mathrm{~g})$ and $1(\mathrm{~h})$, respectively. We analyze them analytically in the framework of the SCTMA; see Supplemental Material [30]. For the 585 defect we find the following equation for the self-energy:

$$
\Sigma=\frac{-\beta n_{\mathrm{imp}}}{(E-\Sigma) \log [-i(E-\Sigma) / t]} .
$$

This form is the result of a divergent zero-energy $T$ matrix [29,30]. Such defects are known as resonant. For the case of the 585 defect $\beta=12.5 \mathrm{eV}^{2}$. In Fig. 2 we compare the spectral weight obtained from direct numerical diagonalization of the disordered lattice model [panels (a)-(c)] with the solution of the self-consistency Eq. (4) [panels (d)-(f)]. For both calculations, the resulting structure near the Dirac point is very similar to experimentally measured graphene dispersion. Stretching of the spectrum near the Dirac point
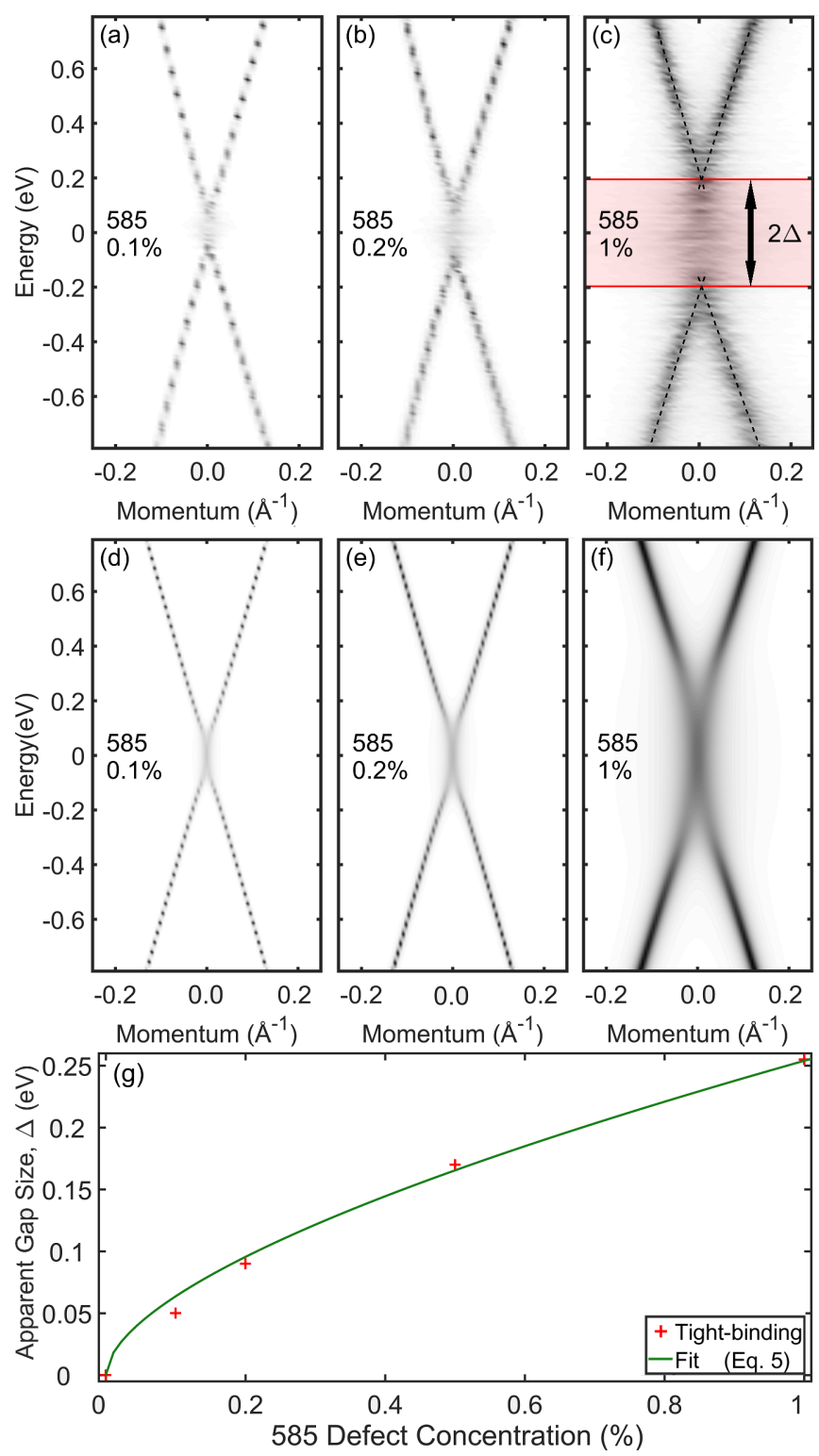

FIG. 2. Band structure of graphene with 585 defects obtained from (a)-(c) exact diagonalization of the tight-binding Hamiltonian and (d)-(f) SCTMA calculation. A significant "stretching" near the Dirac point is already visible for $0.1 \%$ defect density. Such spectrum is typical for resonant impurities. (g) The apparent gap size as a function of defect concentration compared to Eq. (5) (shown with solid line).

can be estimated from Eq. (4) as

$$
\Delta=\sqrt{\frac{2 \beta n_{\mathrm{imp}}}{\left|\log \left(c n_{\mathrm{imp}}\right)\right|}},
$$

where $c \sim 1$ is a fitting parameter. Here $\Delta$, or the apparent gap size, is defined as the absolute value of the energy found at one of the linear crossings in the band dispersion. We plot the size of the smeared region around the Dirac point in Fig. 2(g) along with the fit $(c=2.0712 \pm 0.38)$.

Another type of structural defects [SW, Fig. 1(e)] belongs to the nonresonant case. In this case, the SCTMA provides the 

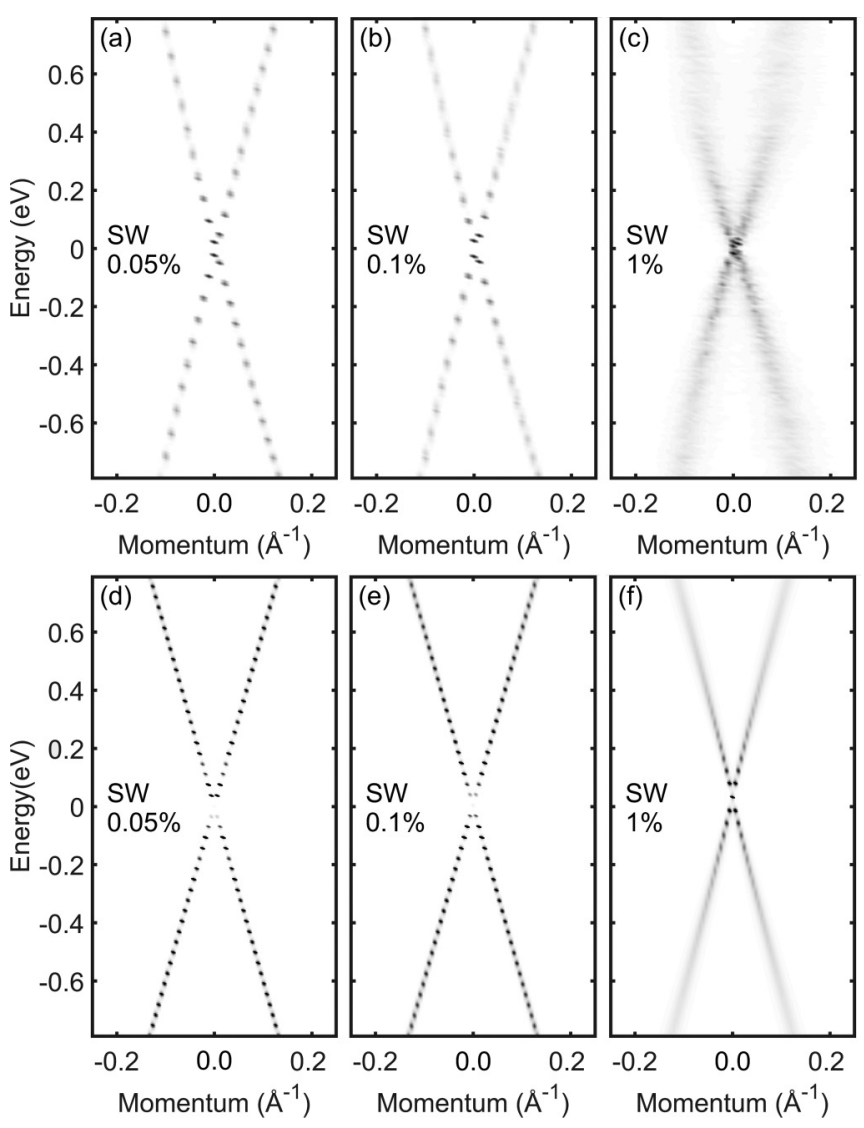

FIG. 3. Electron spectrum of graphene with SW defects obtained from (a)-(c) exact diagonalization of the tight-binding Hamiltonian and (d)-(f) SCTMA calculation. In contrast to the 585 defect, cf. Fig. 2, stretching near the Dirac point does not occur. Instead the linewidth broadening gradually increases away from the Dirac point. This behavior is typical for nonresonant impurities.

following self-energy equation [30]:

$$
\Sigma=n_{\text {imp }}\{\alpha(E-\Sigma) \log [-i(E-\Sigma) / t]\} .
$$

For the SW defect in graphene, we find $\alpha=6.85$. The band dispersion from the direct numerical diagonalization and from the SCTMA are shown in Fig. 3. There is no apparent elongated Dirac point even at relatively high concentrations of impurities. Instead an energy-dependent line broadening gets stronger away from the Dirac point. This is in contrast to the relatively uniform broadening found in the band dispersion with resonant impurities. We see that both for resonant 585 defects and nonresonant SW defects, the results of direct diagonalization and SCTMA calculation show a remarkable agreement.

The two forms of the self-energy, Eqs. (4) and (6), are mathematically the only two possibilities for point defects in graphene (as long as sublattice symmetry is preserved). In the resonant case, the zero-energy $T$ matrix diverges; hence it can be approximated as $T \sim 1 / E$ (up to a logarithmic factor). This leads to the self-consistency equation of the form in Eq. (4). In the nonresonant case, small-energy expansion of the $T$ matrix starts with a nonessential constant (it can be absorbed in the chemical potential) and a linear term leading to Eq. (6). Both resonant and nonresonant cases are
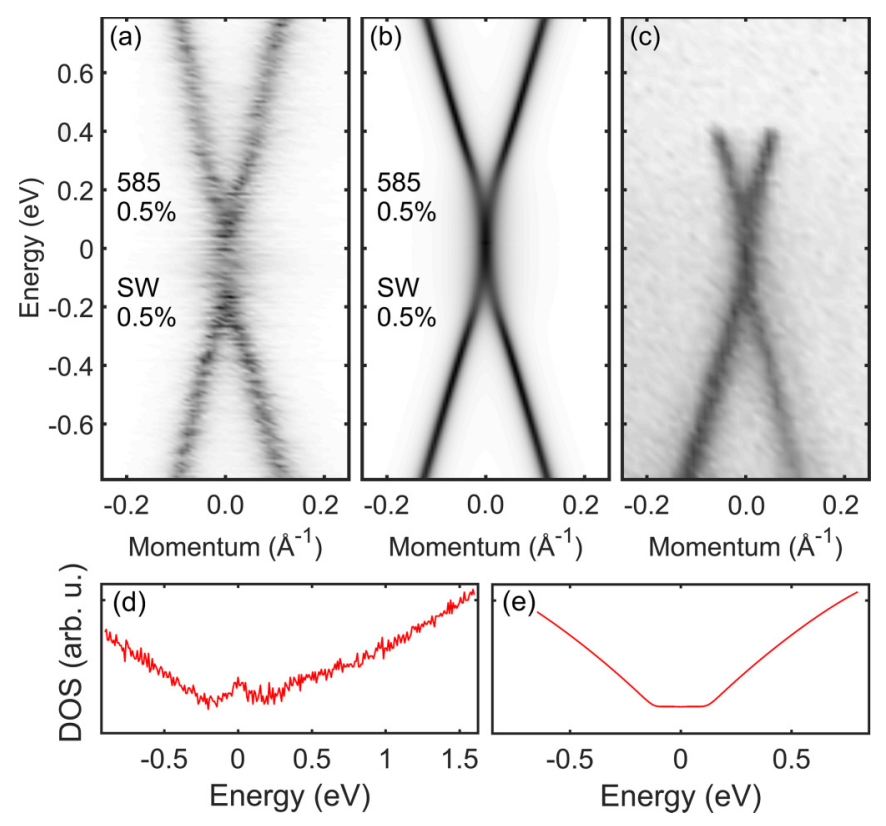

FIG. 4. Electron spectrum of graphene with equal concentration of 585 and SW defects from (a) exact diagonalization of the tightbinding Hamiltonian and (b) SCTMA calculation. Vicinity of the Dirac point is dominated by the resonant 585 impurities with the characteristic stretching of the spectrum; cf. Fig. 2. Linewidth away from the Dirac point is broadened mainly by the weak SW defects as in Fig. 3. (c) ARPES spectrum of epitaxial graphene [23-28]. (d),(e) Average DOS corresponding to the spectra in (a),(b).

in a good agreement with our direct numerical simulations of the tight-binding model. When the sublattice symmetry is broken, self-energy is not a number anymore but rather an operator in the sublattice space. Then more possibilities beyond Eqs. (4) and (6) emerge [33]. Vacancies distributed in only one sublattice, Figs. 1(a) and 1(f), is one possible illustration of this effect.

We also consider a mixture of 585 and SW defects in a single sample to gain insight on how these defects interact and to have an approximation of realistically disordered graphene. In Fig. 4, we compare the band structure for an equal amount of 585 and SW defects obtained from direct diagonalization [panel (a)] and the SCTMA calculation [panel (b)] with the ARPES data of epitaxial graphene [panel (c)] [23-28]. The average DOS is shown in Figs. 4(d) and 4(e) for the tightbinding model and SCTMA, respectively. In the tight-binding model we implement an equal number of 585 and SW defects in the lattice, while for the SCTMA calculation the selfconsistency equation contains the sum of the right-hand sides of Eqs. (4) and (6). The DOS is a momentum integral of the previously calculated spectral weight.

The results in Figs. 4(a) and 4(b) not only show a striking resemblance to each other but also match the "elongation" found in experimentally measured epitaxial graphene dispersions as seen in Fig. 4(c). The DOS shows a nearly constant region near the Dirac point, while further away it matches the linear dispersion of states expected for graphene. This finite DOS near the Dirac point is dominated by the effect from resonant defects whose $T$ matrix diverges at zero energy. The 
DOSs also show an excellent agreement between the SCTMA and the direct tight-binding model.

\section{DISCUSSION}

From our calculations, we can make several conclusions and comment on some new insight. First, the only possibility for a gap in the spectrum is when the sublattice symmetry is broken, for example, in the case of vacancies in a single sublattice, Fig. 1(f). Mathematically, this can be understood by representing the tight-binding Hamiltonian in the matrix form as [40]

$$
H=\left(\begin{array}{cc}
0 & h \\
h^{\dagger} & 0
\end{array}\right)
$$

Here, the block $h$ contains hopping terms from one sublattice to the other and $h^{\dagger}$ defines the opposite hopping. Second nearest-neighbor hopping is temporarily disregarded. Removing atoms from only one sublattice causes $h$ and $h^{\dagger}$ to be nonsquare blocks. The matrix (7) has a number of zero eigenvalues equal (or larger [42]) to the difference of the number of $\mathrm{A}$ and $\mathrm{B}$ sites. Hence the DOS exhibits a delta peak at zero energy with a gap opening around it. This gap is a result of statistical repulsion between zero and nonzero eigenstates of the random Hamiltonian matrix [39,40]. When the second nearest-neighbor hopping is taken into account, the zero-energy eigenstates rearrange into a dispersive midgap band found in Fig. 1(f). These results suggest that selective removal of (or chemical bonding to) carbon atoms from a given sublattice may be the only way to realize gapped graphene experimentally.

The second remark is on the ability to now categorize defects, and about the origin of the elongated Dirac point that has been measured experimentally in epitaxial graphene [23-28]. The SCTMA shows that a point defect in graphene can only be either resonant or nonresonant, where the geometry of a defect affects only the parameters $\alpha$ or $\beta$, effectively giving us a model that can be used to categorize defects in graphene without needing to specify them. Furthermore, due to the remarkable similarity between the experimentally measured elongated Dirac point [Fig. 4(c)], the tight-binding result [Fig. 4(a)], and the SCTMA result [Fig. 4(b)], we infer that this elongation is caused, at least in part, by resonant defects. Although other effects may also contribute to this signature, Fig. 4 suggests that a 585 defect concentration of $0.5 \%$ could explain the elongation we observe. We have shown that 585 defects are resonant and provide an apparent stretching of the Dirac point. At the same time, it is known that 585 defects are common in epitaxial graphene [34,41]. We thus conclude that the elongation of the Dirac point observed in many graphene samples is in part the result of resonant defects which could partially be accounted for by 585 defects. In addition, the resonant and nonresonant defect picture we have demonstrated could provide a way of estimating the percentage of certain types of defects in graphene simply by looking at the stretching of the Dirac point. Regarding the prospective band gap in epitaxial graphene, our calculations explicitly show that there are states in the Dirac point region, leading us to conclude that the elongation of the Dirac point cannot be considered as a gap. Furthermore, any concentration of resonant defects will increase the number of states at low energies. Nevertheless, the stretching of the spectrum near the Dirac point creates an energy range where electrons are localized in real space. This phenomenon may be used to open a mobility gap around the Dirac point in graphene and realize an insulating state [43].

We would also like to comment on a potential outlook resulting from these findings. Primarily, it would be interesting to see how easily extended our model could be to other Dirac materials. Some materials, such as germanene and silicene, are for the intents and purposes of this manuscript identical to graphene and should also have defects that can be categorized into resonant and nonresonant types [44,45]. As both silicene's and germanene's band dispersions have Dirac points, it would be safe to speculate that an elongated Dirac point could also be measured in these materials. For more complex materials, calculating the self-energy of various defects using a $T$-matrix approximation could provide a way of categorizing defects in those materials. This would be particularly interesting to test with three-dimensional Dirac materials, such as $\mathrm{Cd}_{3} \mathrm{As}_{2}$ or $\mathrm{Na}_{3} \mathrm{Bi}[7,9]$.

\section{CONCLUSION}

In summary, we implemented a simple real-space tightbinding model that allowed us to calculate the band dispersion of graphene with defects. We found that a band gap can only be induced when the sublattice symmetry is broken as shown in Fig. 1(f). Looking at more realistic defects, we found that the 585 defect creates an elongated Dirac point (Fig. 2) similar to those found in experimentally measured spectra of epitaxial graphene. Graphene with SW defects has a qualitatively different spectrum without apparent stretching (Fig. 3). We have also developed a SCTMA theory [30], which allowed us to classify point defects as either resonant or nonresonant and showed a remarkable agreement with the direct lattice calculations. We conclude that the experimentally observed elongated Dirac points are partially due to resonant defects (which the 585 defect happens to be) and the contribution to the stretching scales as the square root of their concentration. The SCTMA model also provides further insights into the nature of the broadened states measured in epitaxial graphene, showing that the elongated region cannot be considered a spectral gap. At the same time, disorder can lead to localization of the states near the Dirac point and hence to a mobility gap [43].
[1] S. Agnoli and M. Favaro, J. Mater. Chem. A 4, 5002 (2016).

[2] A.-Y. Lu, S.-Y. Wei, C.-Y. Wu, Y. Hernandez, T.-Y. Chen, T.-H. Liu, C.-W. Pao, F.-R. Chen, L.-J. Li, and Z.-Y. Zhuang, RSC Adv. 2, 3008 (2012).
[3] G. Anagnostopoulos, C. Androulidakis, E. N. Koukaras, G. Tsoukleri, I. Polyzos, J. Parthenios, K. Papagelis, and C. Galiotis, ACS Appl. Mater. Inter. 7, 4216 (2015). 
[4] N. P. Armitage, E. J. Mele, and A. Vishwanath, Rev. Mod. Phys. 90, 015001 (2018).

[5] S. M. Young and C. L. Kane, Phys. Rev. Lett. 115, 126803 (2015).

[6] C. Zhang, Y. Zhang, X. Yuan, S. Lu, J. Zhang, A. Narayan, Y. Liu, H. Zhang, Z. Ni, and R. Liu, Nature (London) 565, 331 (2019).

[7] Z. K. Liu, B. Zhou, Y. Zhang, Z. J. Wang, H. M. Weng, D. Prabhakaran, S.-K. Mo, Z. X. Shen, Z. Fang, X. Dai, Z. Hussain, and Y. L. Chen, Science 343, 864 (2014).

[8] S. M. Young, S. Zaheer, J. C. Y. Teo, C. L. Kane, E. J. Mele, and A. M. Rappe, Phys. Rev. Lett. 108, 140405 (2012).

[9] Z. K. Liu, J. Jiang, B. Zhou, Z. J. Wang, Y. Zhang, H. M. Weng, D. Prabhakaran, S-K. Mo, H. Peng, P. Dudin, T. Kim, M. Hoesch, Z. Fang, X. Dai, Z. X. Shen, D. L. Feng, Z. Hussain, and Y. L. Chen, Nat. Mater. 13, 677 (2014).

[10] Q. D. Gibson, L. M. Schoop, L. Muechler, L. S. Xie, M. Hirschberger, N. P. Ong, R. Car, and R. J. Cava, Phys. Rev. B 91, 205128 (2015).

[11] D. Huertas-Hernando, F. Guinea, and A. Brataas, Phys. Rev. B 74, 155426 (2006).

[12] D. A. Abanin, P. A. Lee, and L. S. Levitov, Phys. Rev. Lett. 96, 176803 (2006).

[13] A. Nishide, A. A. Taskin, Y. Takeichi, T. Okuda, A. Kakizaki, T. Hirahara, K. Nakatsuji, F. Komori, Y. Ando, and I. Matsuda, Phys. Rev. B 81, 041309(R) (2010).

[14] C. Coletti, C. Riedl, D. S. Lee, B. Krauss, L. Patthey, K. von Klitzing, J. H. Smet, and U. Starke, Phys. Rev. B 81, 235401 (2010).

[15] A. Bostwick, J. McChesney, T. Ohta, E. Rotenberg, T. Seyller, and K. Horn, Prog. Surf. Sci. 84, 380 (2009).

[16] A. Bostwick, F. Speck, T. Seyller, K. Horn, M. Polini, R. Asgari, A. H. MacDonald, and E. Rotenberg, Science 328, 999 (2010).

[17] I. Gierz, C. Riedl, U. Starke, C. R. Ast, and K. Kern, Nano Lett. 8, 4603 (2008).

[18] I. Gierz, J. Henk, H. Höchst, C. R. Ast, and K. Kern, Phys. Rev. B 83, 121408(R) (2011).

[19] I. Gierz, M. Lindroos, H. Höchst, C. R. Ast, and K. Kern, Nano Lett. 12, 3900 (2012).

[20] X. Du, I. Skachko, A. Barker, and E. Y. Andrei, Nat. Nanotechnol. 3, 491 (2008).

[21] K. S. Novoselov, Z. Jiang, Y. Zhang, S. V. Morozov, H. L. Stormer, U. Zeitler, J. C. Maan, G. S. Boebinger, P. Kim, and A. K. Geim, Science 315, 1379 (2007).

[22] D. A. Abanin, S. V. Morozov, L. A. Ponomarenko, R. V. Gorbachev, A. S. Mayorov, M. I. Katsnelson, K. Watanabe, T. Taniguchi, K. S. Novoselov, L. S. Levitov, and A. K. Geim, Science 332, 328 (2011).

[23] A. Bostwick, T. Ohta, T. Seyller, K. Horn, and E. Rotenberg, Nat. Phys. 3, 36 (2007).

[24] S. Y. Zhou, G.-H. Gweon, A. V. Fedorov, P. N. First, W. A. De Heer, D.-H. Lee, F. Guinea, A. H. Castro Neto, and A. Lanzara, Nat. Mater. 6, 770 (2007).
[25] E. Rotenberg, A. Bostwick, T. Ohta, J. L. McChesney, T. Seyller, and K. Horn, Nat. Mater. 7, 258 (2008).

[26] A. L. Walter, A. Bostwick, K.-J. Jeon, F. Speck, M. Ostler, T. Seyller, L. Moreschini, Y. J. Chang, M. Polini, R. Asgari, A. H. MacDonald, K. Horn, and E. Rotenberg, Phys. Rev. B 84, 085410 (2011).

[27] D. N. Basov, M. M. Fogler, A. Lanzara, F. Wang, and Y. Zhang, Rev. Mod. Phys. 86, 959 (2014).

[28] S. Y. Zhou, D. A. Siegel, A. V. Fedorov, and A. Lanzara, Physica E 40, 2642 (2008).

[29] P. M. Ostrovsky, I. V. Gornyi, and A. D. Mirlin, Phys. Rev. B 74, 235443 (2006).

[30] See Supplemental Material at http://link.aps.org/supplemental/ 10.1103/PhysRevB.101.235116 for a detailed explanation of the self-consistent $T$ matrix approximation.

[31] K. Kaasbjerg and A. P. Jauho, Phys. Rev. B 100, 241405(R) (2019).

[32] M. Titov, P. M. Ostrovsky, I. V. Gornyi, A. Schuessler, and A. D. Mirlin, Phys. Rev. Lett. 104, 076802 (2010).

[33] P. M. Ostrovsky, M. Titov, S. Bera, I. V. Gornyi, and A. D. Mirlin, Phys. Rev. Lett. 105, 266803 (2010).

[34] F. Banhart, J. Kotakoski, and A. V. Krasheninnikov, ACS Nano 5, 26 (2010).

[35] A. Lherbier, S. M.-M. Dubois, X. Declerck, Y.-M. Niquet, S. Roche, and J.-C. Charlier, Phys. Rev. B 86, 075402 (2012).

[36] J. Schelter, P. M. Ostrovsky, I. V. Gornyi, B. Trauzettel, and M. Titov, Phys. Rev. Lett. 106, 166806 (2011).

[37] K. Kaasbjerg, Phys. Rev. B 101, 045433 (2020).

[38] T. O. Wehling, A. V. Balatsky, M. I. Katsnelson, A. I. Lichtenstein, K. Scharnberg, and R. Wiesendanger, Phys. Rev. B 75, 125425 (2007).

[39] V. Häfner, J. Schindler, N. Weik, T. Mayer, S. Balakrishnan, R. Narayanan, S. Bera, and F. Evers, Phys. Rev. Lett. 113, 186802 (2014).

[40] P. M. Ostrovsky, I. V. Protopopov, E. J. König, I. V. Gornyi, A. D. Mirlin, and M. A. Skvortsov, Phys. Rev. Lett. 113, 186803 (2014).

[41] P. Lauffer, K. V. Emtsev, R. Graupner, T. Seyller, L. Ley, S. A Reshanov, and H. B. Weber, Phys. Rev. B 77, 155426 (2008).

[42] N. Weik, J. Schindler, S. Bera, G. C. Solomon, and F. Evers, Phys. Rev. B 94, 064204 (2016).

[43] Disorder-induced localization of electrons near the Dirac point requires breaking of all symmetries of the Dirac Hamiltonian. This includes not only the chiral sublattice symmetry but also the symmetry between two valleys of the spectrum. For a review of possible localization scenarios see, e.g., P. M. Ostrovsky, I. V. Gornyi, and A. D. Mirlin, Eur. Phys. J. Spec. Top. 148, 63 (2007).

[44] J. Zhao, H. Liu, Z. Yu, R. Quhe, S. Zhou, Y. Wang, C. C. Liu, H. Zhong, N. Han, J. Lu et al., Prog. Mater. Sci. 83, 24 (2016).

[45] A. Acun, L. Zhang, P. Bampoulis, M. Farmanbar, A. van Houselt, A. N. Rudenko, M. Lingenfelder, G. Brocks, B. Poelsema, M. I. Katsnelson et al., J. Phys. Condens. Matter 27, 443002 (2015). 